\title{
ANTS COLONY OPTIMISATION OF A MEASURING PATH OF PRISMATIC PARTS ON A CMM
}

\author{
Slavenko M. Stojadinovic ${ }^{1)}$, Vidosav D. Majstorovic ${ }^{1)}$, Numan M. Durakbasa'), \\ Tatjana V. Sibalija ${ }^{3)}$ \\ 1) University of Belgrade, Department for Production Engineering, Kraljice Marije 16, 11120 Belgrade 35 PF 34, Serbia \\ $\triangle$ sstojadinovic@mas.bg.ac.rs, +381113302438,vidosav.majstorovic@sbb.rs) \\ 2) Vienna University of Technology, Department for Interchangeable Manufacturing and Industrial Metrology, 1060 Wien, \\ Getreidemarkt 9/3113,BA09,Austria (durakbasa@ift.tuwien.ac.at) \\ 3) Metropolitan University, Faculty of Information Technology, Tadeusa Koscuska 63, 11000 Belgrade, Serbia (tsibalija@gmail.com)
}

\begin{abstract}
This paper presents optimisation of a measuring probe path in inspecting the prismatic parts on a CMM. The optimisation model is based on: (i) the mathematical model that establishes an initial collision-free path presented by a set of points, and (ii) the solution of Travelling Salesman Problem (TSP) obtained with Ant Colony Optimisation (ACO). In order to solve TSP, an ACO algorithm that aims to find the shortest path of ant colony movement (i.e. the optimised path) is applied. Then, the optimised path is compared with the measuring path obtained with online programming on CMM ZEISS UMM500 and with the measuring path obtained in the CMM inspection module of Pro/ENGINEER ${ }^{\mathbb{R}}$ software. The results of comparing the optimised path with the other two generated paths show that the optimised path is at least $20 \%$ shorter than the path obtained by on-line programming on CMM ZEISS UMM500, and at least $10 \%$ shorter than the path obtained by using the CMM module in Pro/ENGINEER ${ }^{\mathbb{R}}$.
\end{abstract}

Keywords: path planning, ACO, CMM, prismatic parts.

\section{Introduction}

The Coordinate Measuring Machine (CMM) has been recognised as a powerful tool for dimensional and geometric tolerance inspection in the manufacturing industry [1]. Demands of a competitive, global market imply reduction of the time needed for inspection on a CMM. The inspection time is directly proportional to the length of a measuring probe path, which is influenced by several factors such as complexity of a measuring part (i.e. workpiece), the number of tolerances, quality and type of tolerances, etc. Reduction of the measuring path length results in reduction of the total inspection time, which further reduces the inspection costs and total manufacturing time.

An Ant Colony (AC) algorithm, proposed by M. Dorigo and explained in detail in [2-5], is one of the swarm intelligence methods and widely accepted metaheuristic optimisation techniques. The Travelling Salesman Problem (TSP) is a basis for application of ACO in the coordinate metrology. An example of TSP solution is presented in [6]. Besides ACO, other optimisation techniques have been used to obtain the optimal measuring path, such as a genetic algorithm [7, 8], neural networks [1], and an optimisation algorithm presented in [9]. Data processing for generating the optimal measuring path could be based on the engineering ontology [10-12] and the knowledge base [13], where the knowledge base connects geometry and tolerances of a workpiece. A holistic approach in data definition has been developed [14] to enable the data transfer among different employee groups and departments in an enterprise, 
with the main goal to improve employees' qualifications and enhance the overall product quality. Feature-based inspection planning systems that define initial measuring paths are presented in [15-17]. They are used to depict the workpiece geometry and connect it to the tolerances using the knowledge base. The optimal path of a measuring sensor is generated for the feature that takes part in the tolerance formation. The current status of using geometric tolerances in intelligent manufacturing systems is considered in [18].

Concepts for the automatic inspection planning are presented in [19, 20]; they could serve as a basis for development and implementation of optimisation models. The state of the art in inspection planning is given in [21], whereas trends in development of the production metrology in intelligent manufacturing are shown in [22].

Prismatic Parts (PPs) are an important group of mechanical parts frequently used in industry. PPs are created from the basic geometric features such as plane, cylinder, cone, etc. From the metrological point of view, this group also implies lack of surfaces whose inspection is not strictly required, and that are present mainly due to esthetical or some other reasons. PPs are present in almost all types of manufacturing. In this paper, we focus on the parts with medium and high quality of tolerance precision, which are used to build machine tools.

This paper presents an application of ACO in the coordinate metrology that aims to optimise the measuring path in measurement of PPs on a CMM. The main advantage of ACO in solving the observed problem is its simple implementation based on the developed mathematical model that transforms the problem onto TSP. In particular, inspection of a prismatic part with high geometrical complexity and metrological diversity, produced in small or single quantities, is considered. Besides, automatic generation of the optimal measuring path based on the CAD model presents an advantage in comparison with the existing optimisation methods known from the literature. The developed model can be also applied in hybrid coordinate measuring systems [23], as well as in virtual coordinate measuring machines [24], in order to minimise the inspection time and cost.

The rest of the paper is organised as follows. Section 2 presents the data model; the mathematical and ACO models are presented in Sections 3 and 4, respectively. The optimisation results are shown in Section 5, and the conclusions are contained in Section 6. The basis for ACO model are the data from IGES file of the PP CAD model and the mathematical model that gives a set of points used to find the shortest distance or the optimal path of a measuring probe. The optimal path is compared with the path obtained by on-line programming on CMM ZEISS UMM500 and the path generated in Pro/ENGINEER, using the same parameters. A prismatic part is produced in order to perform this experiment, i.e. to compare the ACO-based optimal path with the paths obtained with the other two methods.

\section{Data model}

A data model developed to apply ACO and to perform comparison with the other two methods is composed of three parts, as presented in Fig. 1. The first part involves the data for development of a measuring path obtained by on-line CMM programming; the second part refers to the data needed to obtain a set of points or initial path and to apply ACO; the third part presents the data for automatic generation of a measuring path in Pro/ENGINEER.

\subsection{Inspection Feature Construction (IFC)}

The IFC data model is based on the basic geometric primitives and their parameters, as presented in [13]. The basic geometric primitives involved in IFC are: point, plane, circle, cylinder, cone, truncated cone, hemisphere, and truncated hemisphere. 


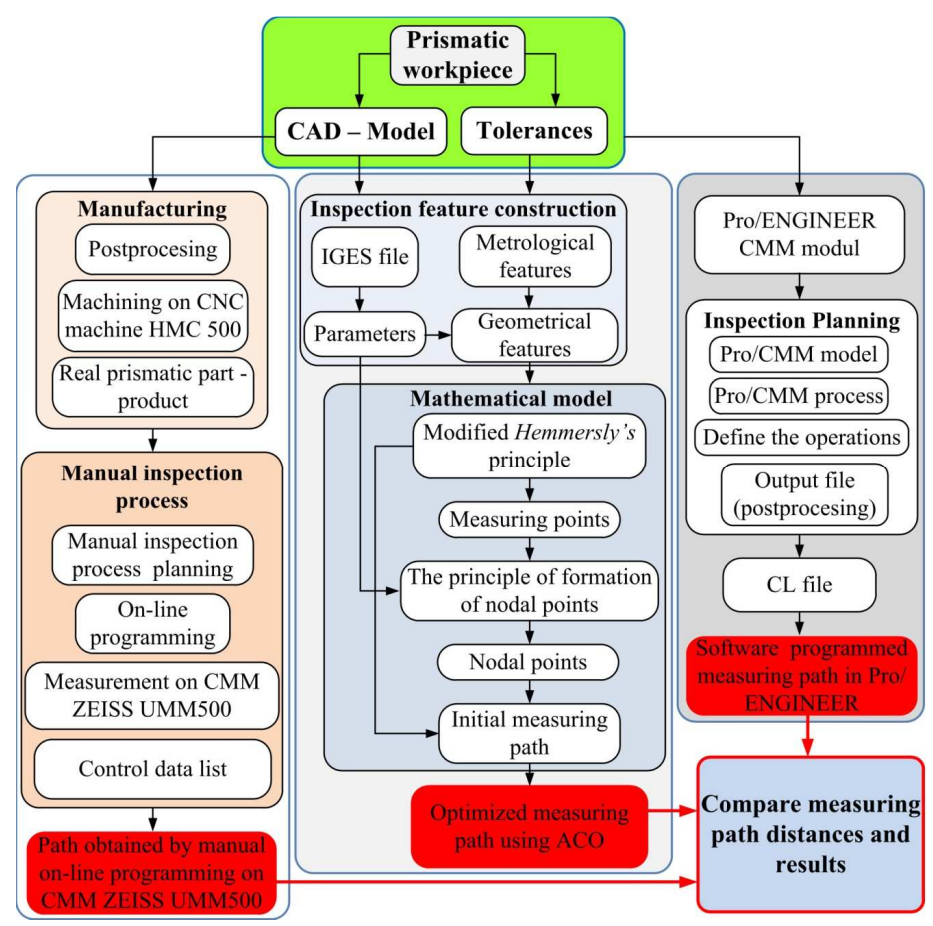

Fig. 1. The data model.

Each geometric primitive is uniquely defined by the coordinate system of a primitive $O_{F}, X_{F}, Y_{F}, Z_{F}$ with respect to the coordinate system of a workpiece $O_{W}, X_{W}, Y_{W}, Z_{W}$, and the set of belonging parameters - with respect to the coordinate system $O_{F}, X_{F}, Y_{F}, Z_{F}$. The meanings of these parameters a presented in Figs. $2 \mathrm{a}$ and $2 \mathrm{~b}$ for two geometric primitives: plane and truncated cone. The parameters can be of the following types: diameter $(D-$ the larger diameter of a cone, $D_{1}$ - the smaller diameter of a cone), height ( $H$ - the height of cone, $H_{1}$ - the height of frustum), width $(a)$, length $(b)$, normal vector of a primitive (n), fullness vector of a primitive $\left(\mathbf{n}_{\mathbf{p}}\right)$. The normal vector $\mathbf{n}$ defines the orientation of a primitive in space. The fullness parameter is determined by the unit vector of $X$-axis of a primitive. The fullness vector $\mathbf{n}_{\mathrm{p}}=\left[\begin{array}{ccc}-1 & 0 & 0\end{array}\right]$ defines a full primitive, whereas the vector $\mathbf{n}_{\mathrm{p}}=\left[\begin{array}{lll}1 & 0 & 0\end{array}\right]$ defines an empty primitive (Fig. 2c). The fullness parameter and the normal vector define the direction of a measuring probe access in generating the measuring path.

a)

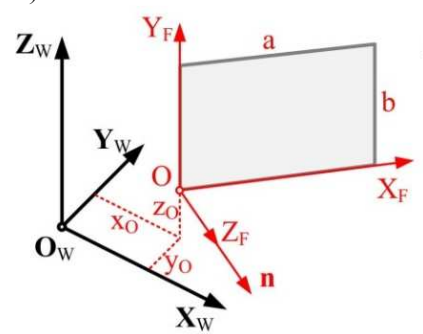

b)

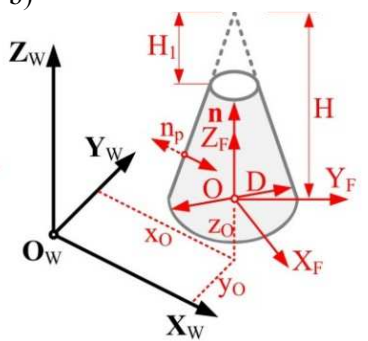

c)

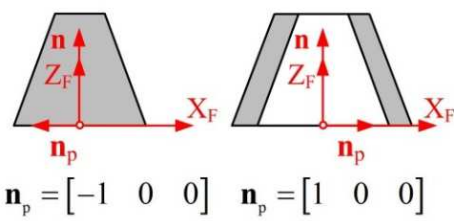

Fig. 2. The geometric primitives: a) plane; b) truncated cone; c) interpretation of fullness vector. 


\subsection{Workpiece machining}

A workpiece, i.e. a prismatic part used for measurement on a CMM and development of the on-line measuring path is produced as follows. Machining of a prismatic part was performed on the 4-axes (X' Y Z B') CNC machining centre (horizontal milling \& drilling machine, type 'LOLA HMC 500'), as presented in Fig. 3. The workpiece characteristics are: dimensions $95 \times 95 \times 95 \mathrm{~mm}$, surface finish quality - N7, material - aluminium. The following tolls were used: end mills, tool-holders for internal cylindrical surfaces, and a spherical milling cutter for hemisphere surfaces. The produced prismatic workpiece contains all geometric primitives mentioned in Subsection 2.1 (enclosed in IFC), which are needed to test the ACO-based model.

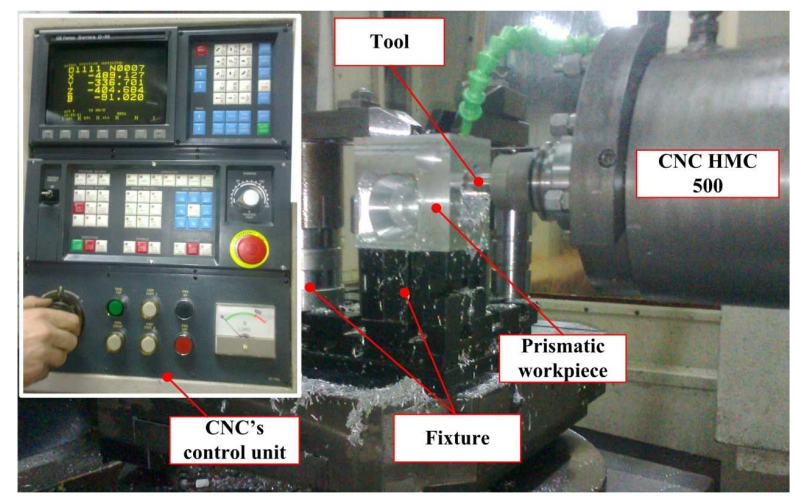

Fig. 3. Machining of a prismatic workpiece on the 4-axes CNC machining centre.

\subsection{On-line programmed measuring path on a CMM}

The experimental installation for the Prismatic Part (PP) measurement on a CMM is shown in Fig. 4. The workpiece measurement was performed in a single clamp; the measuring probe configuration is shown in Fig. 4; the tool used for fixing the PP is a manual vice.

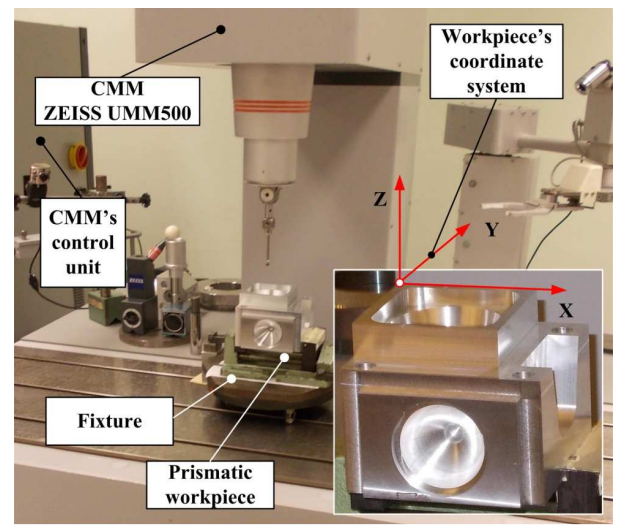

Fig. 4. The experimental installation for the workpiece measurement on a CMM.

The experiment was performed on a CMM ZEISS UMM500, whose main technical characteristics are: a) the number of axis $-3(\mathrm{X}, \mathrm{Y}, \mathrm{Z})$; b) the measuring range $-500 \mathrm{x} 200 \mathrm{x}$ $300 \mathrm{~mm}$; c) MPE $-0.4+\mathrm{L} / 600 \mu \mathrm{m}$; d) accuracy $-0.2 \mu \mathrm{m}$. The inspection process is composed of the preparation and measuring processes. The preparation process involves: 1) mounting the 
workpiece, including the choice of fixing tools and accessories; 2) configuration of the measuring probes; 3 ) calibration of the measuring probes using the calibration sphere; and 4) alignment of the workpiece. In the measuring process, coordinates of the on-line measuring path are taken from the control data list (i.e. STEUERDATENLISTE ZEISS UMESS) for the workpiece. Apart from other data (e.g. the measuring process parameters such as velocity and acceleration), the control list contains the coordinates of points passed by the measuring sensor in generating the on-line programmed measuring path. They are used to obtain the on-line pointto-point measuring path, which is compared with the paths obtained with the other two methods.

The on-line programming method presented in this section is not novel. In this paper, it is used to obtain a measuring path that is compared to the measuring paths obtained by the other two methods.

\subsection{Software programmed measuring path in Pro/ENGINEER ${ }^{\circledR}$}

In order to obtain a measuring path, a module "Manufacturing" and its sub-module "CMM" in Pro/ENGINEER ${ }^{\circledR}$ (version Wildfire 4.0) were used. The coordinate system of a workpiece during the inspection corresponds to the workpiece coordinate system used for inspection on the CMM. Fig. 5 shows the measuring path for inspection of a hemisphere diameter, as well as a part of the generated Cutter Location (CL) file. It is used for verification of the software programmed measuring path in Pro/ENGINEER.

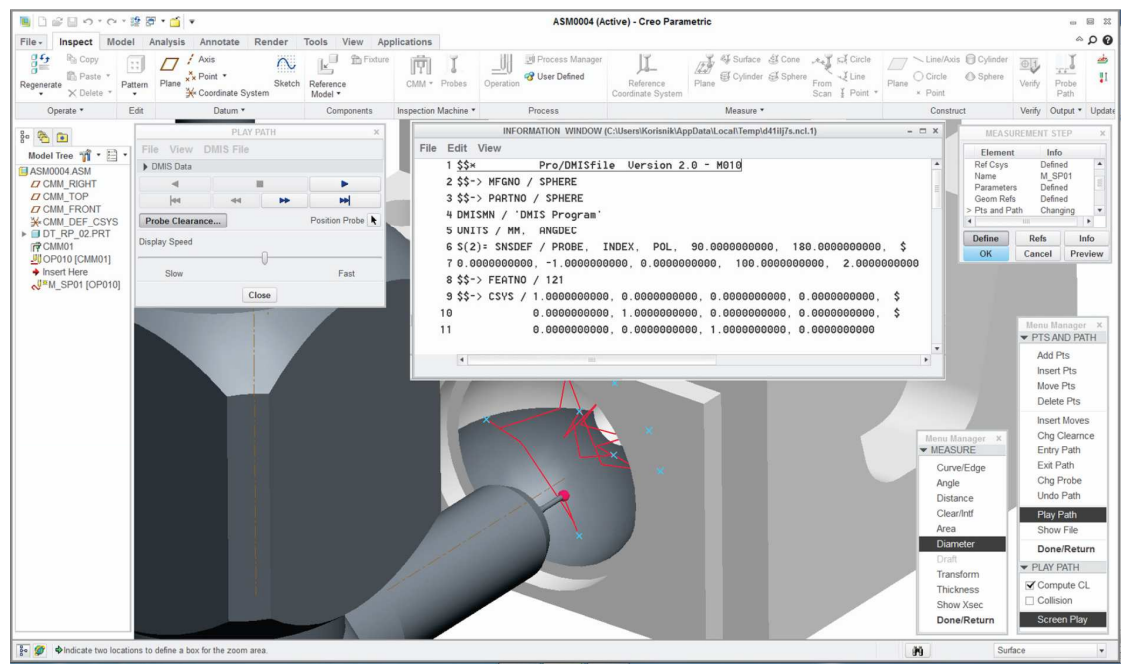

Fig. 5. Inspection of hemisphere in Pro/ENGINEER, with belonging CL file.

The Cutter Location (CL) is a file generated by software as the output report containing the data on the measuring probe motion. The coordinates of points passed by the measuring probe are taken from the CL file, and then the software-programmed point-to-point measuring path is obtained.

To provide a valid comparison between the optimal measuring path and the softwaregenerated measuring path, it is necessary to appropriately tune several parameters in Pro/ ENGINEER, such as APPROACH_DISTANCE, PULLOUT_DIST, MEAS_APPR_DIST, MEAS_PULLOUT_DIST, etc. The values of these parameters must be in accordance with those of the parameters of the mathematical model $\left(d_{1}, d_{2}\right.$, etc.). These settings are performed in the dialog window "Measurement step" in the option "Parameters". 


\section{Mathematical model}

The mathematical model defines distribution of two sets of points: (i) the set of measuring points, and (ii) the set of nodes which is composed of two sub-sets. The mathematical model of the measuring points' distribution is based on the Hemmersly sequences, and used for calculation of coordinates along two axes $s_{i}$ and $t_{i}$ of a feature. The Hemmersly sequences, as specified in [25], define a method for distribution of points, which is applicable for various purposes. The extended version of Hemmersly sequences applied in the coordinate metrology and measurements on CMM, and its comparison with distribution of uniform and random points, is presented by Lee et al. in [26]. In brief, based on the results of research presented in [26], the selected strategy for distribution of measuring points, in comparison with the uniform and random strategies, has a better accuracy. This conclusion is found by comparing Root Mean Square Error (RMSE) for different features.

Distribution of measuring points for different geometric features such as plane, circle, hemisphere, cylinder, etc., is obtained by modifying the Hemmersly sequences. The coordinates of measuring points are presented in the Cartesian coordinate system and named $P_{i}\left(s_{i}, t_{i}, w_{i}\right)$. As an example, the formulas for calculating the coordinates of measuring points for a cylinder are:

$$
\begin{gathered}
s_{i}=R \cos \left(-\frac{\pi}{2}-\frac{2 \pi}{N} \cdot i\right), \\
t_{i}=R \sin \left(-\frac{\pi}{2}-\frac{2 \pi}{N} \cdot i\right), \\
w_{i}=\left(\sum_{j=0}^{k-1}\left(\left[\frac{i}{2^{j}}\right] \operatorname{Mod} 2\right) \cdot 2^{-(j+1)}\right) \cdot h,
\end{gathered}
$$

where: $k=\log _{2} N ; N$ is the desired number of measuring points $i=0,1,2, \ldots,(N-1) ; R \mathrm{~mm}$ is the radius of cylinder and $h \mathrm{~mm}$ is the height of cylinder; Mod2 presents a mathematical operator whose result is the rest from dividing the expression by two. The set of node points implies two sub-sets: $P_{i 1}\left(s_{i 1}, t_{i 1}, w_{i 1}\right)$ and $P_{i 2}\left(s_{i 2}, t_{i 2}, w_{i 2}\right)$, where $i=0,1,2, \ldots,(N-1)$. The sub-set $P_{i 1}$ represents points for the transition from fast to slow feed. The distance that the measuring probe passes between points $P_{i 1}$ and $P_{i}\left(x_{i}, y_{i}, z_{i}\right)$ is represented by $d_{1}-$ the slow feed path, and the distance between points $P_{i 2}$ and $P_{i 1}$ - by $d_{2}$ - the rapid feed path. For inspection of the prismatic parts, there are three different cases of defining the sets $P_{i 1}$ and $P_{i 2}$, as presented in Fig. 6.

a)

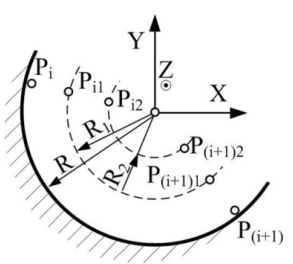

b)

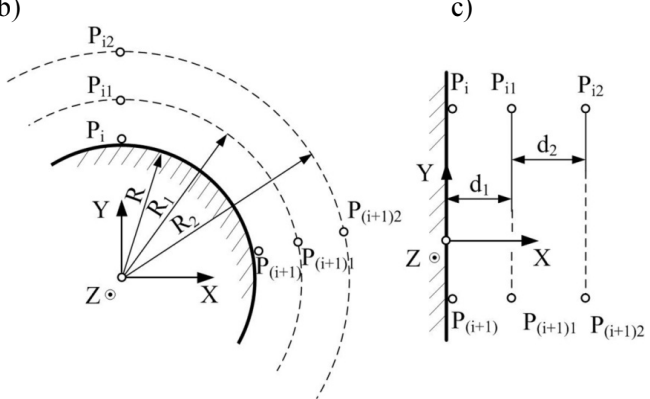

Fig. 6. Distribution of the measuring and node points of a measuring path for the concave, convex and flat surfaces, respectively. 
The main difference in determination of these sets for the concave (Fig. 6a) and convex (Fig. 6b) surface is in the direction of vector $\mathbf{n}_{p}$. For the concave surface this vector is $\mathbf{n}_{p}=\left[\begin{array}{lll}1 & 0 & 0\end{array}\right]$, and for the convex surface $\mathbf{n}_{p}=\left[\begin{array}{lll}-1 & 0 & 0\end{array}\right]$. The procedure implies the following steps:

- Formation of a vector $\overrightarrow{P_{i} O}=\left(x_{0}-x_{i}\right) \vec{i}+\left(y_{0}-y_{i}\right) \vec{j}+\left(z_{0}-z_{i}\right) \vec{k}$, where the centre of a circle is defined by $O\left(x_{0}, y_{0}, z_{0}\right)$;

- Formation of a vector $\mathbf{n}_{p i}=\frac{\overrightarrow{P_{i} O}}{\left|\overrightarrow{P_{i} O}\right|}$;

- Formation of vectors:

$\overrightarrow{P_{i} P_{i 1}}=\overrightarrow{n_{p i}} \cdot d_{1}=x_{P_{i} P_{11}} \vec{i}+y_{P_{i} P_{11}} \vec{j}+z_{P_{i} P_{11}} \vec{k}$ and $\overrightarrow{P_{i} P_{i 2}}=\overrightarrow{n_{p i}} \cdot d_{2}=x_{P_{i} P_{i 2}} \vec{i}+y_{P_{i} P_{i 2}} \vec{j}+z_{P_{i} P_{i 2}} \vec{k}$ (for a concave surface $d_{1}=R-R_{1}$ and $d_{2}=R-R_{2}$, and for a convex surface $d_{1}=R_{1}-R$ and $d_{2}=R_{2}-R$ );

- Calculation of the coordinates:

$$
\begin{aligned}
& x_{i 1}=x_{P_{i} P_{i 1}}+x_{i}, y_{i 1}=y_{P_{i} P_{i 1}}+y_{i}, z_{i 1}=z_{P_{i} P_{11}}+z_{i}, \\
& x_{i 2}=x_{P_{i} P_{i 2}}+x_{i}, y_{i 2}=y_{P_{i} P_{i 2}}+y_{i}, z_{i 2}=z_{P_{i} P_{i 2}}+z_{i} .
\end{aligned}
$$

For a flat surface (Fig. 6c), since the vector $\mathbf{n}$ is taken from IGES file, determination of $P_{i 1}$ and $P_{i 2}$ implies formation of vectors $\overrightarrow{P_{i} P_{i 1}}=\vec{n} \cdot d_{1}=x_{P_{1} P_{11}} \vec{i}+y_{P_{i} P_{1}} \vec{j}+z_{P_{i} P_{11}} \vec{k} \quad$ and $\overrightarrow{P_{i} P_{i 2}}=\vec{n} \cdot d_{2}=x_{P_{i} P_{2}} \vec{i}+y_{P_{i} P_{i 2}} \vec{j}+z_{P_{i} P_{i 2}} \vec{k}$, where constants $d_{1}$ and $d_{2}$ are adopted according to the diameter of measuring probe. Then, the coordinates are calculated using (4).

\section{ACO model}

Development of algorithms for distribution of the measuring points and determination of the collision-free path (presented in [27]) preceded development of an optimisation model using ACO. In contrast to the research presented in [28] that uses the A-star algorithm for determination of the collision-free path, our algorithms give the collision-free points which are used as the input for ACO in order to generate the point-to-point optimised path. Hence, the obtained initial path is a collision-free path. Obstacles, such as edges/borders, holes, etc., can be overcome at two levels, as follows. At the first level, the node points for a single primitive are generated, which assures collision-free inspection of a primitive (e.g. during the hole inspection). The second level implies development of the collision-free points during the motion from one primitive to another, in order to overcome such obstacles, as edges/borders.

The main novelty of the presented research is the way in which the initial path is defined and optimised. Opposite to our research, the path developed in the research [28] is not collisionfree. However, selection of the measuring instrument is not considered in our research, i.e. the path is considered as a set of points with precisely defined sequence.

The measuring path can be presented as a set of points (defined by the mathematical model) passed by the measuring probe during inspection of a primitive. Application of ACO in the coordinate metrology is based on a solution of TSP, where the set of cities visited by the salesman using the shortest possible path corresponds to the set of points of the minimal measuring path length. Precisely, the set of cities corresponds to the set of points, and the salesman corresponds to the measuring probe. Since it is necessary to avoid collision between a workpiece and the measuring probe during measurements on the CMM, the mathematical model must be developed to present distribution of points for basic geometric primitives and for their unique description. 
The model is based on the following equation for calculation of the measuring probe path during the measurement on $N$ measuring points:

$$
D_{t o t}=\sum_{i=0}^{N-1}\left(\left|\overrightarrow{P_{i 2} P_{i 1}}\right|+2 \cdot\left|\overrightarrow{P_{i 1} P_{i}}\right|+\left|\overrightarrow{P_{i 1} P_{(i+1) 2}}\right|\right) \text {. }
$$

The part of a measuring path $2 d_{1}=2 \cdot\left|\overrightarrow{P_{i 1} P_{i}}\right|$, which corresponds to the access (slow movement of the probe) and the return movement of the probe for collision-free inspection, should be excluded from consideration. This is justified since the value $2 d_{1}=2 \cdot\left|\overrightarrow{P_{i 1} P}\right|$ is rather small (up to $10 \mathrm{~mm}$ ), so it does not significantly affect the total value of measuring path.

Therefore, the relation (5) is transformed onto the following relation:

$$
D_{\text {tot }}=K+\sum_{i=0}^{N-1}\left(\left|\overrightarrow{P_{i 1} P_{(i+1) 2}}\right|\right),
$$

where $K=N \cdot\left(2 \cdot d_{1}+d_{2}\right)$ and $d_{2}=\left|\overrightarrow{P_{i 2} P_{i 1}}\right|$.

The relation (6) represents the equation of the initial path and contains the constant and variable parts.

Since the measuring probe does not have to use the variable path $\overrightarrow{P_{i 1} P_{(i+1) 2}}$ every time, the optimisation equation, i.e. the ant colony path, is obtained by using the initially defined path, as follows:

$$
\min \left\{D_{t o t}\right\}=K+\left\{\sum_{i=0}^{N-1}\left(\min \left\{\left|\overrightarrow{P_{i 1} P_{(i+1) 2}}\right|\right\} \vee \min \left\{\left|\overrightarrow{P_{i 1} P_{(i+1) 1}}\right|\right\} \vee \min \left\{\left|\overrightarrow{P_{i(i+1)} P_{(i+1) 2}}\right|\right\} \vee \min \left\{\left|\overrightarrow{P_{i 2} P_{(i+1) 1}}\right|\right\}\right)\right\} .
$$

The difference between the initial path and the ant colony path for the observed three cases (concave surface, convex surface and flat surface, respectively) can be observed in Fig. 7.

a)

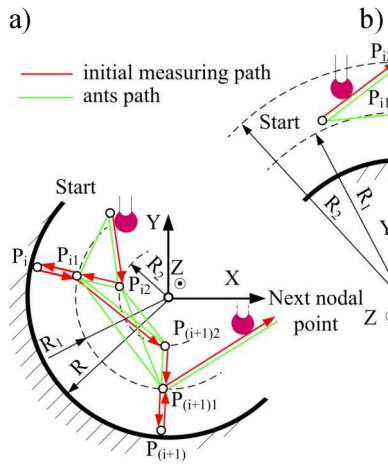

b)

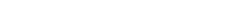
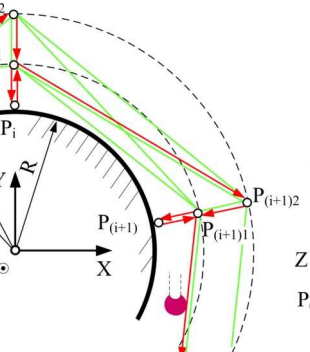

Next noda point c)

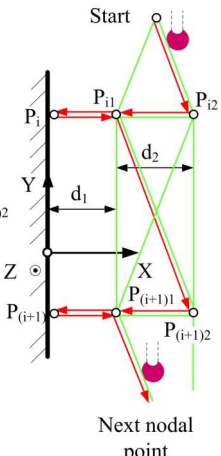

Fig. 7. The initial measuring path and ant the colony path for the concave, convex and flat surfaces, respectively.

\subsection{Travelling Salesman Problem (TSP)}

According to [3] TSP can be represented by a complete weighted graph $G=(N, A)$ with $N$ being the set of nodes representing the cities, and $A$ being the set of $\operatorname{arcs}$. Each $\operatorname{arc}(i, j) \in A$ is assigned a value (length) $d_{i j}$, which is the distance between cities $i$ and $j$, with $i, j \in N$. The goal in TSP is to find the minimum length Hamiltonian circuit of the graph where a Hamiltonian circuit is a closed path visiting each of $n=|N|$ nodes of $G$ exactly once [3], so that the optimal 
solution for TSP is a permutation $\pi$ of the node indices $\{1,2, \ldots, n\}$ such that the length $f(\pi)$ is minimal where $f(\pi)$ is given by:

$$
f(\pi)=\sum_{i=1}^{n-1} d_{\pi(i) \pi(i+1)}+d_{\pi(n) \pi(1)}
$$

\subsection{Tour construction}

The Ant System (AS) technique is used for the tour construction, where $m$ artificial ants concurrently build a TSP-like tour. Ants randomly visit cities, i.e. - in this case - points $P_{i 1}\left(x_{i 1}, y_{i 1}, z_{i 1}\right)$ and $P_{i 2}\left(x_{i 2}, y_{i 2}, z_{i 2}\right)$ defined by the mathematical model. At each construction step, ant $k$ applies a probabilistic action choice rule, called the random proportional rule, to decide which point to visit next. In particular, the probability with which ant $k$, currently at point $i$, chooses to go to point $j$ is:

$$
p_{i j}^{k}=\frac{\left[\tau_{i j}\right]^{\alpha}\left[\eta_{i j}\right]^{\beta}}{\sum_{l \in \mathrm{N}_{i}^{k}}\left[\tau_{i l}\right]^{\alpha}\left[\eta_{i l}\right]^{\beta}}, \text { if } j \in \mathrm{N}_{i}^{k},
$$

where: $\eta_{i j}=\frac{1}{d_{i j}}$ is the heuristic value that is available a priori; $\alpha$ and $\beta$ are parameters which determine the relative influence of the pheromone trail and the heuristic information; $N_{i}^{k}$ is the feasible neighbourhood of ants $k$ when being at point $i$, that is, the set of points that ant $k$ has not visited yet. According to the recommendations for AS technique, the following values are adopted: $\alpha=1$ i $\beta=5$.

\subsection{Updating pheromone trails}

After all the ants have constructed their tours, the pheromone trails are updated. This is done by first lowering the pheromone value on all arcs by a constant factor, and then adding pheromone on the arcs the ants have crossed in their tours. Pheromone evaporation is implemented by:

$$
\tau_{i j} \leftarrow(1-\rho) \tau_{i j}, \forall(i, j) \in L,
$$

where $\rho=0.1$ is the pheromone evaporation rate. The parameter $\rho$ is limited to $0<\rho \leq 1$ and used to avoid unlimited accumulation of the pheromone trails, and it enables the algorithm to "forget" bad decisions previously taken. After evaporation, all ants deposit pheromone on the arcs they have crossed in their tour:

$$
\tau_{i j} \leftarrow \tau_{i j}+\sum_{k=1}^{m} \Delta \tau_{i j}^{k}, \forall(i, j) \in L,
$$

where $\Delta \tau_{i j}^{k}$ is the amount of pheromone deposited by ant $k$ on the arcs it has visited. It is defined as follows:

$$
\Delta \tau_{i j}^{k}=\left\{\begin{array}{cc}
1 / C^{k}, & \text { if } \operatorname{arc}(i, j) \text { belongs to } T^{k} \\
0, & \text { otherwise }
\end{array}\right\},
$$

where $C^{k}$ is the length of the tour $T^{k}$ built by ant $k$, computed as the sum of the lengths of arcs belonging to $T^{k}$. 


\section{Optimisation results}

The results of optimisation can be analysed by comparing the lengths of three observed measuring paths, as shown in Fig. 8, where the on-line programmed path is blue, the software generated path is green and the optimised path is red.

As it has been already mentioned, in order to perform a credible comparison between the online programmed path and the optimal path it is necessary to set up the parameters in the same way for both methods. The parameters of primitives for the observed prismatic workpiece are: a) plane: $a=95 \mathrm{~mm}, b=59 \mathrm{~mm}, N=4, d_{1}=2.6666 \mathrm{~mm}, d_{2}=8 \mathrm{~mm}$; b) circle: $R=22,5 \mathrm{~mm}$, $N=4, d_{1}=3 \mathrm{~mm}, d_{2}=9 \mathrm{~mm}$; c) truncated hemisphere: $R=20 \mathrm{~mm}, H_{1}=0,65 \mathrm{~mm}, N=8$, $d_{1}=1,6 \mathrm{~mm}, d_{2}=8 \mathrm{~mm}$; d) cylinder: $R=17,5 \mathrm{~mm}, H=20 \mathrm{~mm}, N=8, d_{1}=1,4 \mathrm{~mm}, d_{2}=7 \mathrm{~mm}$; e) truncated cone: $R=27 \mathrm{~mm}, H=76 \mathrm{~mm}, H_{1}=56 \mathrm{~mm}, N=12, d_{1}=2,16 \mathrm{~mm}, d_{2}=10,8 \mathrm{~mm}$. These values are selected according to the limitation of the manufacturing and measuring resources used in this experiment (i.e. characteristics of $\mathrm{CNC}, \mathrm{CMM}$, tools, fixtures, probes, etc.).

Analysis of the measuring process parameters such as velocity and acceleration is not considered in this paper. In the presented approach the analysis is based on the length of measuring paths obtained by three methods (the on-line programmed measuring path on the $\mathrm{CMM}$, the software generated measuring path, and the optimised path obtained with ACO). Thus, the measuring process parameters can be adjusted in the same way for all three methods used to generate the measuring path.

In the ACO application, to obtain the optimal path the ant colony with $m=500$ ants was used in 100 iterations.

In order to present the comparison of three methods and the optimisation results (Table 1), the following variables are considered: $d_{m}$ is the measuring path length obtained by on-line programming on CMM ZEISS UMM500; $d_{s}$ is the measuring path length obtained in Pro/ENGINEER; $d_{o}$ is the optimized measuring path length obtained with ACO. A realistic comparison is assured by the basic postulate that the measuring process is the same in all three cases (methods), which can be adjusted so that the parameters $\left(d_{1}, d_{2}\right.$, , APPROACH DISTANCE, PULLOUT_DIST, MEAS_APPR_DIST, MEAS_PULLOUT_DIST, etc.) have the same value in all three methods. The influence of the start and end points is minimised using such a setting that the parameters of measuring process are the same in all three methods. Regarding the start point defined by the points' distribution method, the measuring sensor makes a move from a distance (defined by the parameters) toward it in the direction perpendicular to the measuring surface. Hence, the path length generated by the measuring sensor before reaching the start point is not included in the total measuring path length. The end point is the last measuring point and its coordinates depend on distribution of the selected points, which should not be influenced in order to provide a realistic comparison.

The length of the path generated by on-line programming $\left(d_{m}\right)$ is taken from the control data list on CMM ZEISS UMM500, the software measuring path length $\left(d_{s}\right)$ is taken from the corresponding CL file generated in Pro/ENGINEER, while the optimized path length $\left(d_{0}\right)$ is the shortest path obtained with the ACO-based model. The software path is obtained by analysing the CL file that shows points used for construction of the measuring path.

Table 1 shows the values of measuring path lengths obtained with three methods $\left(d_{m}, d_{s}\right.$ and $d_{o}$ ), as well as the results of comparison. For the observed primitives (i.e. features), the ratios of measuring path lengths $d_{o} / d_{s}$ are less than $87,19 \%$, which presents reduction of the measuring path obtained with ACO by at least $12,81 \%$. The ratios of measuring path lengths $d_{o} / d_{m}$ are less than $78,40 \%$, presenting reduction of the measuring path by at least $21,60 \%$. In accordance to MPE, the results of comparisons were rounded to $1 \mu \mathrm{m}$. 

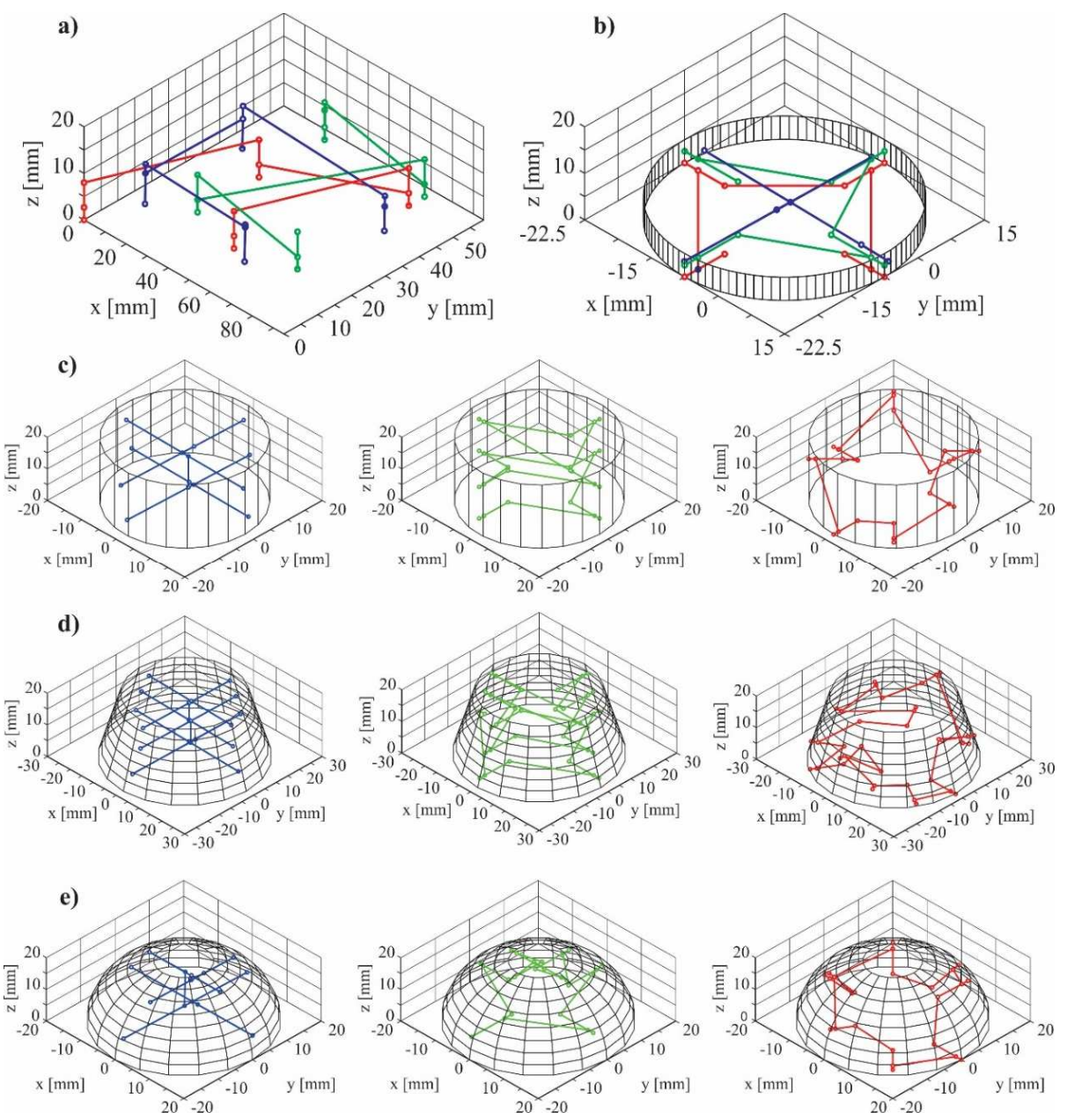

Fig. 8. The results and comparison of three obtained measuring paths for geometric primitives: a) plane; b) circle; c) cylinder; d) truncated cone; e) truncated hemisphere

Table 1. Comparison of the measuring path lengths obtained with three methods.

\begin{tabular}{|l|l|l|l|l|l|}
\hline \multirow{2}{*}{ Results } & \multicolumn{5}{|c|}{ Features } \\
\cline { 2 - 6 } & Plane & Circle & Truncated hemisphere & Cylinder & Truncated cone \\
\hline$d_{m} \mathrm{~mm}$ & 203.390 & 154.354 & 244.858 & 290.984 & 507.037 \\
\hline$d_{s} \mathrm{~mm}$ & 202.652 & 126.442 & 183.676 & 228.987 & 440.140 \\
\hline$d_{o} \mathrm{~mm}$ & 159.460 & 110.246 & 159.096 & 172.214 & 307.609 \\
\hline$d_{o} / d_{s} \%$ & 78.69 & 87.19 & 86.62 & 75.21 & 69.89 \\
\hline$d_{o} / d_{m} \%$ & 78.40 & 71.42 & 64.97 & 59.18 & 60.67 \\
\hline
\end{tabular}

It has been well known in the coordinate metrology that the length of a measuring path is directly proportional to the measuring time. Therefore, the obtained reduction in the optimised path lengths directly reduces the total time of inspection of a prismatic workpiece on the CMM. The advantage of the proposed model is better exposed when there is a large number of tolerances in a workpiece, and in the case of high geometric complexity of a prismatic workpiece for medium and high precision of tolerances. 


\section{Conclusion}

The paper presents AC-based optimisation of a measuring path in prismatic part inspection on a CMM. The optimisation is based on a previously developed mathematical model for distribution of measuring and node points of a measuring path, and on a solution of TSP obtained by using ACO.

The optimal measuring path is compared with the on-line programmed measuring path on a CMM and with the automatically generated measuring path in Pro/ENGINEER (the CMM module), for the observed prismatic workpiece. Comparison between the optimal path obtained with ACO and the on-line programmed path shows improvement, i.e. reduction of the measuring path length by more than $20 \%$. The optimal path obtained with ACO is over $10 \%$ shorter that the path obtained with Pro/ENGINEER, using the same parameter settings for both methods.

Besides the mentioned results, the advantage of ACO is its simple implementation based on the developed mathematical model which transforms a measuring path onto a set of points and converts the optimisation problem onto TSP. The output of AC-based optimisation is the optimised point-to-point measuring path for measurement of the basic geometric primitives. Similarly to CNC programming (such as G-code), the obtained output can be used for off-line programming on a CMM, where the optimised path is given in a point-to-point form.

The presented research has been conducted within the scope of wider research that refers to development of an intelligent system for inspection planning of PPs, and aims to address main industrial demands such as high geometrical variability of modern products and fast delivery, i.e. short time-to-market. The overall goals are: (i) reduction of the total manufacturing time, where the presented research contributes in terms of reduction of the inspection time, and (ii) improvement of the inspection quality through automation of the activities that are usually performed by an inspection planner.

Since this model is developed only for the basic geometric primitives, the main limitation of the presented research refers to its application for prismatic parts only, i.e. the proposed model cannot be used for the inspection of free-form surfaces.

Evaluation of the measuring uncertainty for the developed model can be modelled using a virtual measuring machine model presented in [29] and ISO 15530-3 [30]. The prerequisite condition is adaptation, i.e. modification, of a module that simulates the residual and random errors dependent on the kinematic system of CMM, according to the working space (i.e. the measuring range of $500 \times 300 \times 200$ ) and the main structure type.

\section{Acknowledgment}

The presented research was supported by the Ministry of Education, Science and Technological Development of the Republic of Serbia and Vienna University of Technology, Department for Interchangeable Manufacturing and Industrial Metrology, High Precision Measurement Room - Nanometrology Laboratory.

\section{References}

[1] Hwang, Y.C., Tsai, Y.C., Chang, A.C. (2004). Efficient inspection planning for coordinate measuring machines. Int. J. Adv. Manuf. Technol., 23,732-742.

[2] Dorigo, M., Gambardella, M.L. (1997). Ant colonies for the travelling salesman problem. BioSystems, 43, 73-81.

[3] Dorigo, M., Stützle, T. (2004). Ant Colony Optimization. The MIT Press Cambridge, Massachusetts London, England. 
[4] Blum, C. (2005). Ant colony optimization: Introduction and recent trends. Physics of Life Reviews, 2, 353373.

[5] Dorigo, M., Blum, C. (2005). Ant colony optimization theory: A survey. Theoretical Computer Science, 344, 243-278.

[6] Cheng, F.T., Chun W.T., Ching C.T. (2004). A new hybrid heuristic approach for solving large traveling salesman problem. Information Sciences, 166, 67-81.

[7] Lu, C.G, Morton, D., Wu, M.H., Myler, P. (1999). Genetic algorithm modelling and solution of inspection path planning on a Coordinate Measuring Machine (CMM). Int. J. Adv. Manuf. Technol., 15, 409-416.

[8] Liangsheng, Q., Guanhua, X., Guohua, W. (1998). Optimization of the measuring path on a coordinate measuring machine using genetic algorithms. Measurement, 23, 159-170.

[9] Moroni, G., Petro, S. (2013). Inspection strategies and multiple geometric tolerances. Procedia CIRP, 10, 54-60.

[10] Pellitero, M.S., Barreiro, J., Cuesta, E., Alvarez, J.B. (2011). A new process-based ontology for KBE system implementation: application to inspection process planning. Int. J. Adv. Manuf. Technol., 57, 325-339.

[11] Stojadinovic, S., Majstorovic, V. (2012). Towards the development of feature - based ontology for inspection planning system on CMM. Journal of Machine Engineering, 12(1), 89-98.

[12] Stojadinovic, S., Majstorovic, V. (2014). Developing engineering ontology for domain coordinate metrology. FME Transactions, 42(3), 249-255.

[13] Majstorovic, D.V., Stojadinovic, M.S., Sibalija, V.T. (2015). Development of a knowledge base for the planning of prismatic parts inspection on CMM. Acta IMEKO, 4(2), 10-17.

[14] Weckenmann, A., Werner, T. (2010). Holistic qualification in manufacturing metrology by enhancing knowledge exchange among different user groups. Metrol. Meas. Syst., 17(1), 17-26.

[15] Myeong, W.C., Honghee, L., Gil, S.Y., Jinhwa, C. (2005). A feature - based inspection planning system for coordinate measuring machines. Int. J. Adv. Manuf. Technol., 26, 1078-1087.

[16] Kramer, R.T., Huang, H., Messina, E., Proctor, M.F., Scott, H. (2001). A feature - based inspection and machining system. Computer-Aided Design, 33(9), 653-669.

[17] Kamrani, A., Nasr, A.E., Ahmari, A.A., Abdulhameed, O., Mian, H.S. (2014). Feature-based design approach for integrated CAD and computer-aided inspection planning. Int. J. Adv. Manuf. Technol., 76(912), 2159-2183.

[18] Lemu, G.H. (2014). Current status and challenges of using geometric tolerance information in intelligent manufacturing systems. Adv. Manuf., 2, 13-21.

[19] Yuewei, B., Shuangyu, W., Kai, L., Xiaogang, W. (2010). A Strategy to Automatically Planning Measuring Path with CMM Offline. Proc. of International Conference on Mechanic Automation and Control Engineering (MACE), Wuhan, China, 3064-3067.

[20] Hussien, A.H., Youssefy, M.A., Shoukryz, K.M. (2012). Automated Inspection Planning system for CMMs. Proc. of International Conference on Engineering and Technology, Cairo, Egypt, 1-6.

[21] Zhao, F., Xu, X., Xie, Q.S. (2009). Computer - aided inspection planning - the state of the art. Computer in Industry, 60(7), 453-466.

[22] Osanna, P.H. (1997). Intelligent production metrology - A powerful tool for intelligent manufacturing. $e \& i$ Elektrotechnik und Informationstechnik, 114, 162-168.

[23] Sładek, J., Sitnik, R., Kupiec, M., Błaszczyk, P. (2010). The hybrid coordinate measurement system as a response to industrial requirements. Metrol. Meas. Syst., 17(1), 109-118.

[24] Sładek, J., Gąska, A., Olszewska, M., Kupiec, R., Krawczyk, M. (2013). Virtual coordinate measuring machine built using lasertracer system and spherical standard. Metrol. Meas. Syst., 20(1), 77-86.

[25] Hammersley, J.M. (1960). Monte Carlo methods for solving multivariate problems. Ann. New York Acad. Sei., 86, 844-874.

[26] Lee, G., Mou, J., Shen, Y. (1997). Sampling strategy design for dimensional measurement of geometric features using coordinate measuring machine. Int. J. Mach. Tools. Manufact., 37(7), 917-934. 
[27] Stojadinovic, S., Majstorovic, V., Durakbasa, N. (2015). A feature - based path planning for inspection prismatic parts on CMM. Proc. of XXI IMEKO World Congress Measurement in Research and Industry, Prague, Czech Republic.

[28] Schmitt, R., Zheng, H., Zhao, X., König, N., Coelho, R.R. (2009). Application of ant colony optimization to Inspection Planning. Proc. of International Conference on Computational Intelligence for Measurement Systems and Applications, Hong Kong, China.

[29] Sładek, J., Gąska, A. (2012). Evaluation of coordinate measurement uncertainty with use of virtual machine model based on Monte Carlo method. Measurement, 45, 1564-1575.

[30] ISO/TS 15530-3: Geometrical product specifications (GPS) - coordinate measuring machines (CMM): Technique for determining the uncertainty of measurement - Part 3, Use of calibrated workpieces or standards (2011). 